\title{
Cyclic AMP signalling pathway and trehalase activation in the fission yeast Schizosaccharomyces pombe
}

\author{
Dolores Carrillo, Jeronima Vicente-Soler and Mariano Gacto
}

Author for correspondence: Mariano Gacto. Tel: +34 68307 100. Fax: +34 68363963.

Department of Genetics and Microbiology, Facultad de Biologia, University of

Murcia, 30071 Murcia, Spain

\begin{abstract}
The response of derepressed cells of Schizosaccharomyces pombe to the addition of glucose included a marked and reversible activation of neutral trehalase that was not produced in repressed cells. The protein synthesis inhibitor cycloheximide, the protonophore 2,4-dinitrophenol or the uncoupler sodium azide also enhanced trehalase activity in derepressed cells provided glucose was present in the incubation assays. However, only 2,4-dinitrophenol or cycloheximide was able to induce trehalase activation in repressed cells. Stimulation of trehalase by these compounds was preceded in all cases by a rapid increase in adenosine 3'-5'-cyclic monophosphate (CAMP) content. Since exogenous CAMP can activate trehalase both in repressed and derepressed growing cells, the results provide evidence for the existence of an induced CAMP signalling pathway in the fission yeast with several entries for trehalase activation. The correlation between CAMP increase and trehalase activation was not maintained when the enzyme was heat-shock-activated, supporting the concept that trehalase activity can be also enhanced in cells by another mechanism in which cAMP does not act as second messenger.
\end{abstract}

Keywords: Schizosaccharomyces pombe, trehalase activation, cyclic AMP, signalling pathways

\section{INTRODUCTION}

The metabolic response of derepressed cells of Saccharomyces cerevisiae to glucose involves a cyclic AMP (cAMP) signalling pathway which has been the subject of considerable attention (for reviews, see Thevelein, 1991, 1992). Genetic and biochemical evidence indicates that $\mathrm{R} A S 1$ and $\mathrm{R} A S 2$ gene products, which are the yeast homologues of the mammalian RAS proteins, mediate a transient activation of adenylate cyclase in response to stimulation by glucose (Gibbs \& Marshall, 1989). The increased level of cAMP promotes binding of the nucleotide to the regulatory subunit of cAMP-dependent protein kinase causing its dissociation from the catalytic subunits which are thus activated. Further transmission of the cAMP signal involves covalent modification by phosphorylation of various key enzymes in carbon metabolism (Wills, 1990).

Among fungi, the existence of glucose-induced cAMP signalling has been reported in several zygomycetes (Dewerchin \& van Laere, 1984; van Mulders \& van Laere, 1984) and suspected in budding yeasts related to $S$. cerevisiae (Arguelles \& Gacto, 1985). In particular, the occurrence of this signal in the fission yeast Schizosaccharomyces pombe has been questioned because there is clear evidence that the single RAS protein present in this yeast does not act as a regulator of adenylate cyclase (Fukui et al., 1986; Thevelein, 1991). However, recent results by Byrne \& Hoffman (1993) provide evidence for a glucose-induced cAMP signalling pathway in this yeast that appears to be required for glucose repression of the $f b p 1$ gene.

In this context, previous studies from this laboratory have shown that the addition of fermentable sugars, protonophores or uncouplers to derepressed cells of $S_{c}$. pombe results in activation of the neutral trehalase (EC 3.2.1.28) and that this stimulation is an energy-requiring posttranslational event which can also be triggered both in repressed and derepressed growing cells by adding cAMP to the incubation buffer (Carrillo et al., 1992). In the present report, we show that the increase in trehalase activity which occurs after addition of the above inducers is indeed preceded by a rapid increase in intracellular cAMP. The role of cAMP in the modulation of trehalase activity in cells under heat shock treatment has also been examined. 


\section{METHODS}

Yeast strains and growth conditions. The wild-type strain Schizosaccharomyces pombe $927 \mathrm{~h}^{-}$was cultured at $28^{\circ} \mathrm{C}$ with shaking in a liquid medium containing $2 \%(\mathrm{w} / \mathrm{v})$ glucose and $0 \cdot 3 \%$ yeast extract. The cells were collected by centrifugation from derepressed stationary-phase cultures after glucose exhaustion $\left(\mathrm{OD}_{600}=4 \cdot 0\right)$. Alternatively, the organism was grown on $2 \%(\mathrm{w} / \mathrm{v})$ glycerol and $0.6 \%$ yeast extract and harvested from derepressed cultures in the exponential phase of growth $\left(\mathrm{OD}_{600}=1 \cdot 0\right)$. The cells were washed with cold distilled water and resuspended in $10 \mathrm{mM} \mathrm{MES} / \mathrm{KOH}$ buffer, $\mathrm{pH} \mathrm{6.0,} \mathrm{at} \mathrm{a}$ concentration of $50 \mathrm{mg}$ wet weight $\mathrm{ml}^{-1}$ (approximately $8 \times$ $10^{8}$ cells $\mathrm{ml}^{-1}$ ). In comparative experiments Saccharomyces cerevisiae $\mathrm{X} 2180-1 \mathrm{~A}$ was cultured in the glucose medium described above, the cultures recovered at stationary phase $\left(\mathrm{OD}_{600}=5 \cdot 0\right)$ and the cells obtained and processed as indicated.

Activation of trehalase and preparation of cell extracts. Suspensions of derepressed cells were incubated in a shaking water bath and allowed to equilibrate at $30^{\circ} \mathrm{C}$ for $5 \mathrm{~min}$. Before and after addition of the compounds assayed (zero time), samples $(1.5 \mathrm{ml})$ were removed at timed intervals and the cells washed as above. To each final pellet, $0.7 \mathrm{ml} 10 \mathrm{mM}$

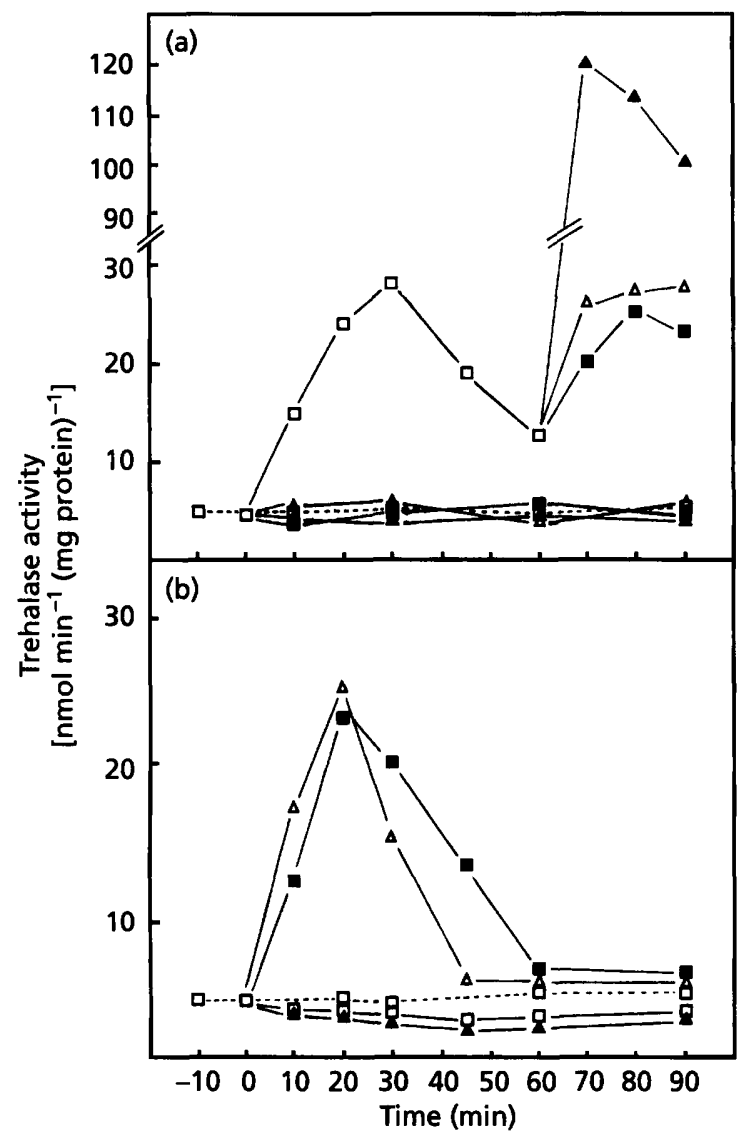

Fig. 1. Evolution of trehalase activity in (a) derepressed and (b) repressed cells upon addition of various compounds. Cells were supplemented at zero time with either $100 \mathrm{mM}$ glucose $(\square)$,

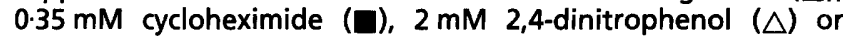
$2 \mathrm{mM}$ sodium azide ( $\Delta)$. Re-additions to derepressed cells supplemented with glucose were carried out 60 min later, when the content of glucose remaining in the cell suspension was $12 \mathrm{mM}$. Basal levels of trehalase in control cells are represented by the discontinuous line.
MES/KOH buffer, $\mathrm{pH} 6.0$, was added and the cells were broken using a vortex mixer with glass beads $(0.5 \mathrm{~mm}$ diameter $)$ and intermittent cooling in an ice bath. The cell extracts were then centrifuged in the cold for $5 \mathrm{~min}$ at $12000 \mathrm{~g}$ in an Eppendorf microfuge and the resulting supernatant fluids were used for enzyme assays and protein determination. In the case of experiments with repressed cells, to ensure repressive conditions, cultures growing exponentially on glucose $\left(\mathrm{OD}_{600}=0.2\right)$ were directly supplemented with the compound to be assayed instead of performing the addition to the cell suspension maintained in buffer. At the time stated in the text, samples $(70 \mathrm{ml})$ were obtained and the cells were washed and mechanically disrupted as indicated for derepressed cells.

For activation of trehalase upon heat shock, the procedure of De Virgilio et al. (1990) was followed. Exponential-phase cultures $\left(\mathrm{OD}_{600}=0.2\right)$ growing on glucose medium were shifted at $40^{\circ} \mathrm{C}$, whereas parallel control cultures were maintained at $28^{\circ} \mathrm{C}$.

Trehalase assay and protein determination. Reaction mixtures for trehalase assay contained $0.6-0.9 \mathrm{mg}$ protein, $100 \mathrm{mM}$ trehalose and $10 \mathrm{mM} \mathrm{MES} / \mathrm{KOH}, \mathrm{pH} 6.0$, in a final volume of $0.5 \mathrm{ml}$. After incubation for $10-30 \mathrm{~min}$ at $30^{\circ} \mathrm{C}$ the reaction tubes were immersed in a boiling water bath for $2 \mathrm{~min}$, cooled and centrifuged at $3000 \mathrm{~g}$ for $10 \mathrm{~min}$. Glucose was determined in the supernatant fluids as described elsewhere (Arguelles \& Gacto, 1985). One unit of trehalase released $1 \mathrm{nmol}$ of glucose min $^{-1}$ under these conditions. Protein content was determined according to the Lowry method.

CAMP determination. cAMP levels and trehalase activity were always determined simultaneously on the same cultures. At the times indicated before and after addition of the various compounds assayed, $250 \mu$ of the cell suspension (about $2 \times 10^{8}$ cells) was withdrawn and transferred into precooled tubes containing the same volume of $1 \mathrm{M}$ trichloroacetic acid (TCA). The samples were immediately frozen in liquid nitrogen, freezethawed four times and then centrifuged in the cold at $12000 \mathrm{~g}$ for $3 \mathrm{~min}$. The supernatants were extracted three times with 4 vols water-saturated ethyl ether to eliminate TCA from the extracts and thereafter they were freeze-dried, dissolved in $50 \mu \mathrm{l}$

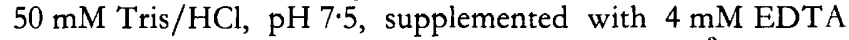
and assayed for cAMP content using the Amersham $\left({ }^{3} \mathrm{H}\right)$ cAMP radioassay kit according to the supplier's instructions. cAMP concentrations were determined within the recommended range of maximum precision and expressed as pmol ( $\mathrm{g}$ wet weight $)^{-1}$. When this same procedure of cAMP determination was applied to $S$. cerevisiae X2180-1A for comparative purposes, the values obtained after addition of glucose were comparable to those described by Arkinstall et al. (1991) for several strains of this yeast.

Reproducibility of results. All experiments were repeated at least three times with similar results. Representative results are shown.

\section{RESULTS}

We have shown previously that addition of glucose to derepressed cells of Sc. pombe provokes a reversible activation of neutral trehalase (Carrillo et al., 1992). In contrast, the addition of the inhibitor of protein synthesis cycloheximide, the protonophore 2,4-dinitrophenol or the uncoupler sodium azide to derepressed cells did not cause, by itself, changes in trehalase activity (Fig. 1a). However, each of these compounds produced a marked stimulation of enzyme activity in the presence of glucose 


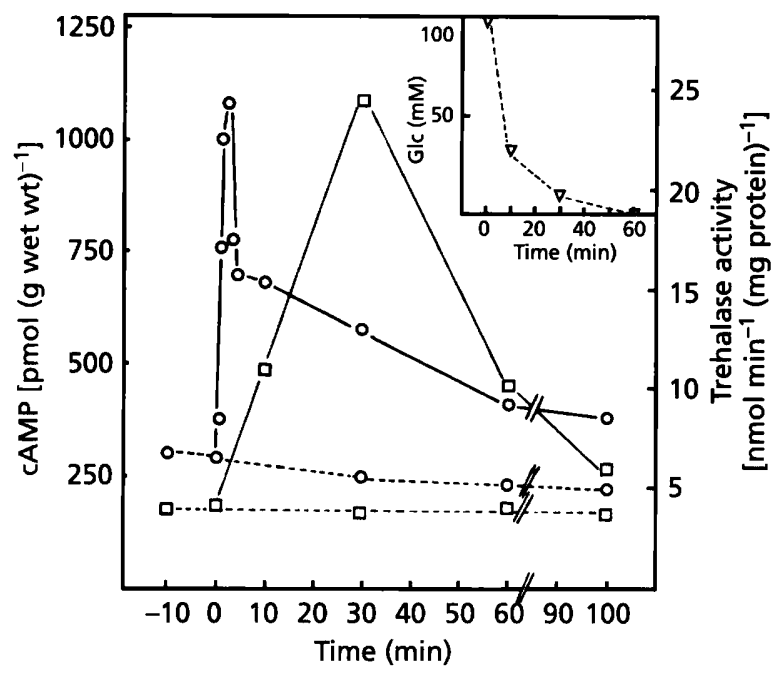

Fig. 2

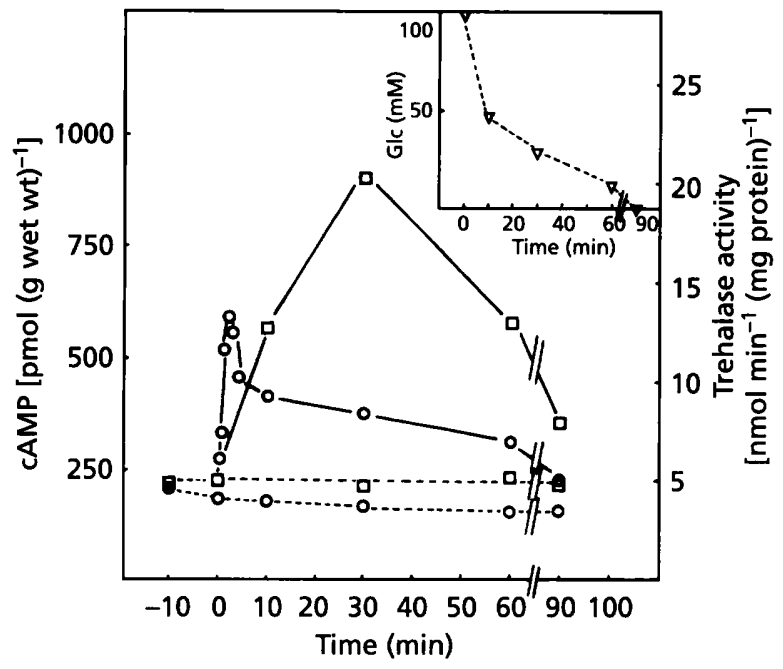

Fig. 3

Fig. 2. Effect of the addition of $100 \mathrm{mM}$ glucose on trehalase activity $(\square)$ and CAMP levels $(O)$ in derepressed cells grown on glycerol. Glucose was added at zero time and trehalase and CAMP determined as described in Methods. The inset shows the consumption of the glucose (Glc) added to the cell suspension. Trehalase and cAMP in control cells are represented by the discontinuous line.

Fig. 3. Effect of the addition of $100 \mathrm{mM}$ glucose to derepressed cells from stationary phase cultures grown on glucose. Symbols for trehalase, CAMP and glucose consumption are as indicated in the legend to Fig. 2.

and was able to reactivate trehalase after a first activationdeactivation process induced by the sugar (Fig. 1a).

When similar experiments were performed with repressed cells, the activity of the trehalase enzyme in cultures supplemented with glucose was almost identical to that of control cultures, indicating that glucose was unable to increase trehalase activity in repressed cells (Fig. 1b). Confirming earlier results by De Virgilio et al. (1991), we found about the same basal values for neutral trehalase activity during exponential growth on glucose and in stationary phase (Fig. 1a, b) so that the lack of activation of trehalase in repressed cells cannot be interpreted as due to a previous full activation maintained by glucose. The enzyme activity also was unaffected in repressed cells by sodium azide, whereas after addition of cycloheximide or 2,4-dinitrophenol an increase was noticed (Fig. 1b).

In $S$. cerevisiae trehalase is regulated by cAMP-dependent protein phosphorylation (Uno et al., 1983). Since the existence of a cAMP signalling pathway in Sc. pombe has been recently proposed (Hoffman \& Winston, 1991; Byrne \& Hoffman, 1993), it seemed worthwhile to examine possible changes in the intracellular cAMP content under the different conditions in which trehalase was activated in these cells. The results indicated that the addition of glucose to derepressed cells of the fission yeast causes a rapid and pronounced increase in the intracellular level of cAMP prior to trehalase stimulation. This effect was clearly shown both in cells from cultures growing on glycerol (Fig. 2) and in cells from stationary-phase cultures after growth on glucose (Fig. 3). In contrast to what has been described for the budding yeast $S$. cerevisiae

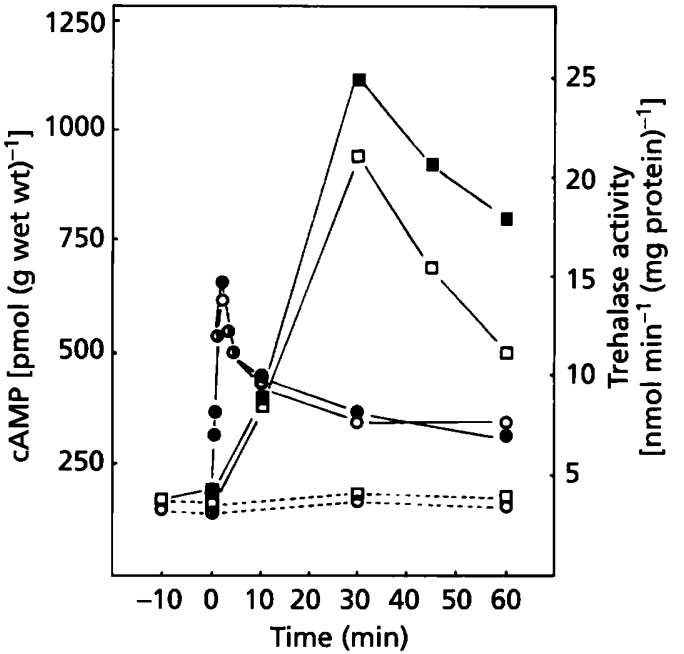

Fig. 4. Evolution of trehalase activity $(\square, \square)$ and CAMP levels $(0,0)$ in derepressed cells from stationary phase cultures grown on glucose upon addition of $100 \mathrm{mM}$ glucose in the presence (filled symbols) or absence (open symbols) of $2 \mathrm{mM}$ acridine orange. When present, the drug was added to the cell suspension $10 \mathrm{~min}$ in advance of the glucose (zero time). Trehalase and CAMP levels in control cells from suspensions not supplemented with glucose are represented by the discontinuous line.

(Thevelein \& Beullens, 1985) neither the glucose-induced cAMP signal nor the subsequent activation of the trehalase enzyme were abolished by the presence of acridine orange in the fission yeast (Fig. 4). On the other hand, the addition of sodium azide to derepressed cells 


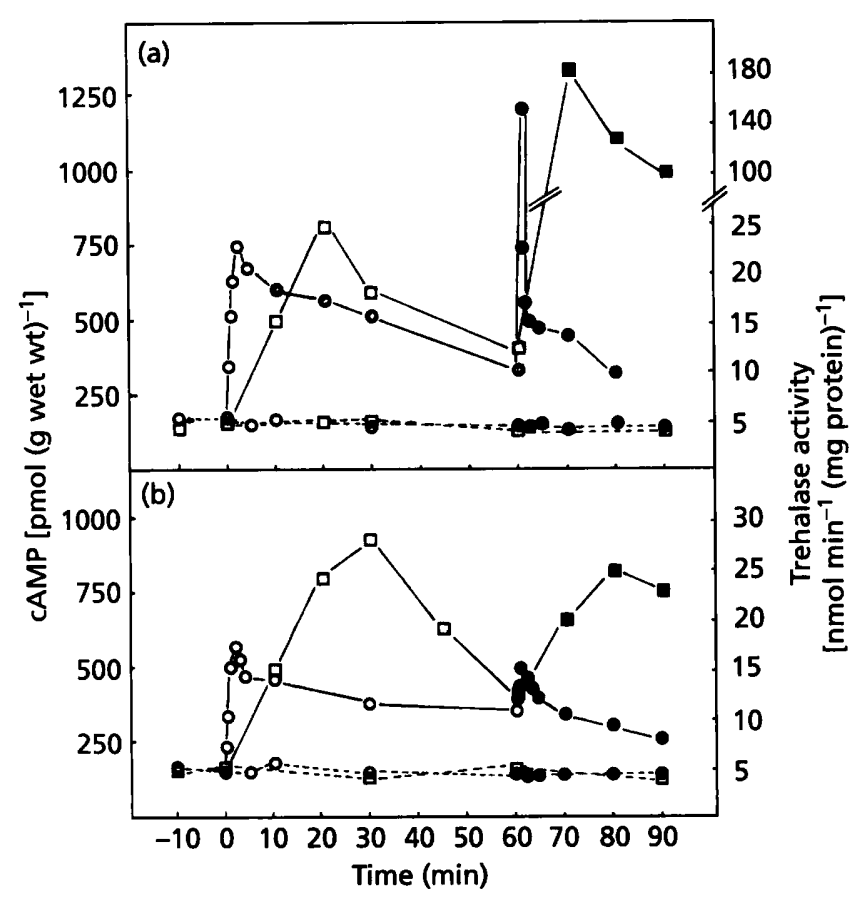

Fig. 5. Changes in trehalase activity $(\square, \square)$ and corresponding levels of $\operatorname{cAMP}(0,0)$ upon addition at zero time of $200 \mathrm{mM}$ glucose to a suspension of derepressed cells from stationaryphase cultures (open symbols) and subsequent re-addition $60 \mathrm{~min}$ later (filled symbols) of $2 \mathrm{mM}$ sodium azide (a) or $0.35 \mathrm{mM}$ cycloheximide (b). At the time of the re-additions the glucose content was about $30 \mathrm{mM}$ in both cell suspensions. Trehalase and CAMP levels in control cells are represented by the discontinuous line.

under conditions which trigger trehalase activation (i.e. with glucose present in the incubation buffer) was also accompanied by a rise in the cAMP level preceding the activation of the enzyme (Fig. 5a). A comparatively smaller, but reproducible increase in cAMP also was detected when cycloheximide was added as an alternative inducer (Fig. 5b). Remarkably, both the peak in cAMP and the activation of trehalase were absent when these compounds were added in the absence of glucose. Similar results were obtained when 2,4-dinitrophenol was used to induce trehalase activation (not shown).

When repressed cells of $S c$. pombe growing on glucosecontaining medium were further supplemented with glucose or sodium azide, no changes in the basal level of cAMP were observed, indicating that not only trehalase activation but also the cAMP pulse promoted by these inducers is sensitive to glucose repression. This result supports the concept of a common link between the two glucose-induced effects. However, within seconds of adding cycloheximide or 2,4-dinitrophenol to repressed cultures, the cells showed increases in cAMP content which were comparable to those illustrated in Fig. 5. Thus, a close correlation between increase in cAMP content and trehalase activation was also established in repressed cells.

The activation of neutral trehalase in heat-shocked cells of

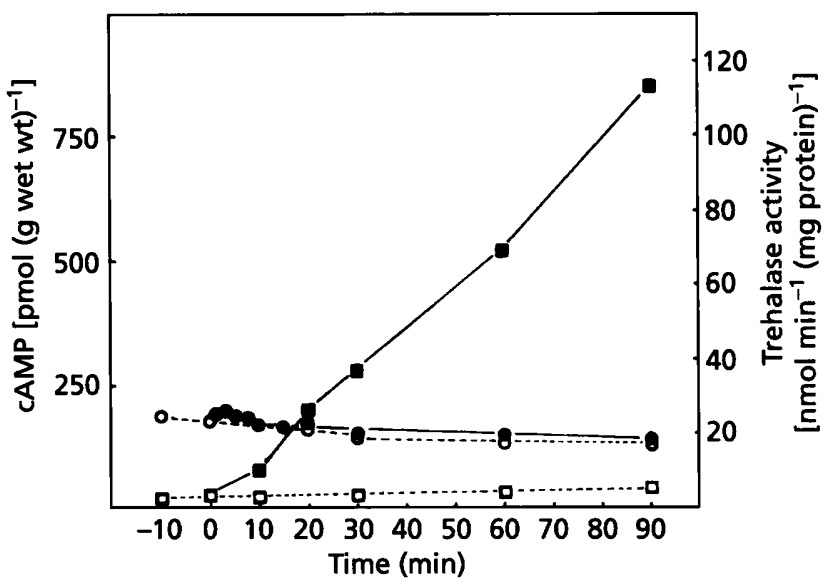

Fig. 6. Trehalase activity $(\square, \square)$ and corresponding CAMP levels $(0,0)$ in repressed cells during a heat shock. Exponentialphase cultures growing on glucose were shifted at zero time to $40^{\circ} \mathrm{C}$ (filled symbols) or maintained at $28^{\circ} \mathrm{C}$ as a control (open symbols). Trehalase and CAMP levels in control cells are represented by the discontinuous line.

Sc. pombe maintained under repression conditions has been described previously (De Virgilio et al., 1990, 1991). In view of the above precedents, we next examined a possible correlation between cAMP increase and trehalase activation under these conditions. As indicated in Fig. 6, repressed cells growing exponentially on glucose markedly increased trehalase activity for at least $90 \mathrm{~min}$ upon exposure of the culture to $40^{\circ} \mathrm{C}$. However, no changes in cAMP content were observed suggesting the existence of an alternative, cAMP-independent pathway responsible for heat shock activation of trehalase (Fig. 6).

\section{DISCUSSION}

In $S_{c}$. pombe cAMP has been implicated in glucoserepression of transcription of the $f b p 1$ gene which encodes fructose-1,6-bisphosphatase (Hoffman \& Winston, 1991). This previous evidence, and more recent results published while this study was in progress (Byrne \& Hoffman, 1993), suggest the existence in this yeast of a cAMP signalling pathway independent of ras and working by activation of cAMP-dependent protein kinase. The measurement of cAMP levels performed by us in derepressed cells immediately after glucose addition supports the conclusion of Byrne \& Hoffman (1993) although, the alteration in cAMP content in response to glucose was a much more rapid event in our hands. In addition, our present work shows that, as in the budding yeast $S$. cerevisiae, the rapid and reversible increase in the cAMP content can be achieved not only by glucose, but also by the inhibitor of protein synthesis cycloheximide, the protonophore 2,4-dinitrophenol or the uncoupler sodium azide which share the ability to trigger trehalase activation. The link between the changes in cAMP induced by these compounds and the parallel, although temporally retarded, activation of trehalase does not stand merely on circumstantial evidence. Taken together with results 
reported earlier, demonstrating that exogenously added cAMP can activate trehalase (Carrillo et al., 1992), our data clearly indicate that glucose, and other inducers, trigger a cAMP signalling pathway which in turn can produce trehalase activation as a final effect. To our knowledge this is the first direct indication of a cAMP signal in the fission yeast able to play a post-translational role by modulating an enzyme activity.

We found that cells grown under repressed and derepressed conditions possess similar steady-state levels of cAMP (see Figs 5 and 6 for basal contents). This finding agrees with results presented by Hoffman \& Winston (1991) and differs strongly from earlier data from Schlanderer \& Dellweg (1974) which indicated a lower cAMP content in repressed cells. In any case, our data on the analysis of the response to glucose in both types of cells reveals differential behaviour. Only derepressed cells show altered cAMP levels and trehalase activation in response to glucose, whereas repressed cells lack the glucose-induced, transient cAMP signal and the subsequent trehalase activation.

We have previously demonstrated that the effect of exogenous cAMP on trehalase activation is not sensitive to glucose repression (Carrillo et al., 1992). In contrast to the cAMP-induced response, the glucose-induced trehalase activation appears to be under glucose catabolite repression. These results point to the existence of some glucose-repressible protein along the signalling pathway implicated in the mechanism of trehalase activation induced by glucose. Thus, the cAMP signalling pathway in $S c$. pombe appears similar in design to that of $S$. cerevisiae, where the participation of a glucose-repressible protein in the glucose-induced signal has been suspected (Thevelein, 1991, 1992). Sodium azide, in contrast to cycloheximide or 2,4-dinitrophenol, does not trigger the cAMP signal in repressed cells although, as with glucose, it does have a triggering effect in derepressed cells (Fig. 1). Most likely, this compound activates the signalling pathway in an analogous way to glucose by interacting with some initial factor upstream of the putative glucose-repressible protein. On the contrary, both cycloheximide and 2,4dinitrophenol, which apparently do not require the function of such an element, would activate a common part of the sensing system downstream of the glucoserepressive protein. This would explain their ability to activate trehalase even in repressed cells. Hence, as in budding yeasts (Thevelein, 1991, 1992), the results support the outline of a branched cAMP signalling pathway leading to trehalase activation throughout several different entries. The fact that, in all cases, the cAMP rise and the corresponding enzyme activation provoked by these compounds required the simultaneous presence of glucose probably indicates the need for available ATP as substrate for adenylate cyclase. In this context, it should be mentioned that $S c$. pombe lacks the glyoxylate cycle and therefore the cells show a greatly reduced ATP content in the absence of glucose or an alternative energy source (McDonald \& Tsai, 1989).

Sc. pombe differs profoundly from $S$. cerevisiae in many biochemical aspects (Russell \& Nurse, 1986). In the fission yeast, for instance, RAS proteins do not act as regulators of adenylate cyclase (Fukui et al., 1986). Moreover, in spite of structural analogies (Yamawaki-Kataoka et al., 1989), functional differences between the adenylate cyclases in both yeasts have been described (Engelberg et al., 1990). Thus, it would not be surprising if, besides a common final effect and the participation of a glucose-repressive protein and cAMP in the two cases, the modulation of trehalase by glucose signal transduction would follow a somewhat different pathway in the two yeasts. Our results on the effect of acridine orange, which inhibits both the cAMP signal and the glucose-induced trehalase activation in the budding yeast (Thevelein \& Beullens, 1985), but not in Sc. pombe, are likely to reflect some peculiarity in the composition of the pathway present in the fission yeast additional to this RAS-independent character. A particularly intriguing question to be resolved is the nature of the event that gives rise to trehalase activation by this pathway. The simplest hypothesis to explain this would be a direct phosphorylation of the enzyme protein by cAMP-dependent protein kinase, as in the case of $S$. cerevisiae (Uno et al., 1983; Ortiz et al., 1983). Results supporting the idea that the glucose-induced trehalase stimulation in Sc.pombe is in fact due to a post-translational covalent modification of the enzyme have been presented previously (Carrillo et al., 1992). However, as reported by others (De Virgilio et al., 1991), we also have been unable to induce in vitro activation of trehalase under conditions in which trehalases from $S$. cerevisiae and Candida utilis become phosphorylated and thereby activated (Ortiz et al., 1983; Arguelles \& Gacto, 1985). In addition, treatment of trehalase from the fission yeast with alkaline phosphatase does not result in changes in enzyme activity (unpublished results). Thus, although the activation appears to be mediated by cAMP-dependent protein kinase, the exact requirements for trehalase modification by this pathway remain unknown.

From our results on the activation of trehalase by heat shock (Fig. 6), it can be deduced that such a response does not follow the same pathway that is functional in the glucose response. No peak in the acid-extractable cAMP pool is produced by thermal treatment prior to the stimulation of trehalase, suggesting that the mechanism which modifies trehalase activity takes place by means of a signal in which CAMP may not be involved as a second messenger. Hence, in $S_{c}$. pombe the cAMP signalling pathway appears to exist in parallel to other pathways that can transmit the same final effect of trehalase activation. A similar situation has been shown recently for nutrientinduced activation of neutral trehalase in starved cells of S. cerevisiae (Hirimburegama et al., 1992). The demonstration in the fission yeast of several pathways controlling trehalase, and probably other enzymes, raises new questions related to the physiological relevance of these responses, the identification of key components and the interaction among the different elements involved in such pathways.

\section{ACKNOWLEDGEMENT}

The skillful assistance of F. Garro is gratefully acknowledged. 


\section{REFERENCES}

Arguelles, J. C. \& Gacto, M. (1985). Evidence for regulatory trehalase activity in Candida utilis. Can J Microbiol 31, 529-537.

Arkinstall, S. J., Papasavvas, S. G. \& Payton, M. A. (1991). Yeast $\alpha$-mating factor receptor-linked G-protein signal transduction suppresses Ras-dependent activity. FEBS Lett 284, 123-128.

Byrne, S. M. \& Hoffman, C. S. (1993). Six git genes encode a glucose-induced adenylate cyclase activation pathway in the fission yeast Schizosaccharomyces pombe. J Cell Sci 105, 1095-1100.

Carrillo, D., Vicente-Soler, J. \& Gacto, M. (1992). Activation of neutral trehalase by fermentable sugars and cAMP in the fission yeast Schizosaccharomyces pombe. FEMS Microbiol Lett 98, 61-66.

De Virgilio, C., Simmen, U., Hottiger, T., Boller, T. \& Wiemken, A. (1990). Heat shock induces enzymes of trehalose metabolism, trehalose accumulation, and thermotolerance in Schizosaccharomyces pombe, even in the presence of cycloheximide. FEBS Lett 273, 107-110.

De Virgilio, C., Muller, J., Boller, T. \& Wiemken, A. (1991). A constitutive, heat-shock activated neutral trehalase occurs in Schizosaccharomyces pombe in addition to the sporulation-specific acid trehalase. FEMS Microbiol Lett 84, 85-90.

Dewerchin, M. A. \& Van Laere, A. J. (1984). Trehalase activity and cyclic AMP content during early development of Mucor rouxii spores. J Bacteriol 158, 575-579.

Engelberg, D., Poradosu, E., Simchem, G. \& Levitzki, A. (1990). Adenylyl cyclase activity of the fission yeast Scbizosaccharomyces pombe is not regulated by guanyl nucleotides. FEBS Lett 261, 413-418.

Fukui, Y., Kozasa, T., Kajiro, Y., Takeda, T. \& Yamamoto, M. (1986). Role of a ras homologue in the life cycle of Schizosaccharomyces pombe. Cell 44, 329-336.

Gibbs, J. B. \& Marshall, M. S. (1989). The ras oncogene-an important regulatory element in lower eucaryotic organisms. Microbiol Rev 53, 171-185.

Hoffman, C. S. \& Winston, F. (1991). Glucose repression of transcription of the Schizosaccharomyces pombe fbp1 gene occurs by a cAMP signalling pathway. Genes \& Dev 5, 561-571.

Hirimburegama, K., Durnez, P., Keleman, J., Oris, E., Vergauwen, R., Mergelsberg, H. \& Thevelein, J. M. (1992). Nutrient-induced activation of trehalase in nutrient-starved cells of the yeast
Saccharomyces cerevisiae: cAMP is not involved as second messenger. $J$ Gen Microbiol 138, 2035-2043.

McDonald, I. J. \& Tsai, C. S. (1989). Continuous culture and intermediary carbon metabolism. In Molecular Biology of the Fission Yeast, pp. 367-396. Edited by A. Nasim, P. Young \& B. F. Johnson. New York: Academic Press.

Ortiz, C. H., Maia, J. C. C., Tenan, M. R., Braz-Padrao, G. R., Mattoon, G. R. \& Panek, A. D. (1983). Regulation of yeast trehalase by a monocyclic, cyclic AMP-dependent phosphorylationdephosphorylation cascade system. J Bacteriol 153, 644-651.

Russell, P. \& Nurse, P. (1986). Schizosaccharomyces pombe and Saccharomyces cerevisiae: a look at yeast divided. Cell 45, 781-782.

Schlanderer, G. \& Dellweg, H. (1974). Cyclic AMP and catabolite repression in yeasts. Eur J Biochem 49, 305-316.

Thevelein, J. M. (1991). Fermentable sugars and intracellular acidification as specific activators of the RAS-adenylate cyclase signalling pathway in yeast: the relationship to nutrient-induced cell cycle control. Mol Microbiol 5, 1301-1307.

Thevelein, J. M. (1992). The RAS-adenylate cyclase pathway and cell cycle life control in Saccharomyces cerevisiae. Antonie Leeunenboek, $J$ Microbiol, special issue Molecular Biology of Yeasts. Edited by L. Grivell. 62, 109-130.

Thevelein, J. M. \& Beullens, M. (1985). Cyclic AMP and the stimulation of trehalase activity in the yeast Saccharomyces cerevisiae by carbon sources, nitrogen sources and inhibitors of protein synthesis. J Gen Microbiol 131, 3199-3209.

Uno, I., Matsumoto, K., Adachi, K. \& Ishikawa, T. (1983). Genetic and biochemical evidence that trehalase is a substrate of cAMP. dependent protein kinase in yeast. $J$ Biol Chem 258, 10867-10872.

Van Mulders, R. M. \& Van Laere, A. J. (1984). Cyclic AMP, trehalase and germination of Pbycomyces blakesleeanus spores. $J$ Gen Microbiol 130, 541-547.

Wills, C. (1990). Regulation of sugar and ethanol metabolism in Saccharomyces cerevisiae. Crit Rev Biochem Mol Biol 25, 245-280.

Yamakawi-Kataoka, Y., Tamaoki, T., Choe, H. R., Tanaka, H. \& Kataoka, T. (1989). Adenylate cyclases in yeast: a comparison of the genes from Scbizosaccbaromyces pombe and Saccharomyces cerevisiae. Proc Natl Acad Sci US A 86, 5693-5697.

Received 28 September 1993; revised 30 November 1993; accepted 17 December 1993. 